\title{
Nanosized cationic hydrogels for drug delivery: preparation, properties and interactions with cells
}

\author{
Serguei V. Vinogradov, Tatiana K. Bronich, Alexander V. Kabanov* \\ Department of Pharmaceutical Sciences, University of Nebraska Medical Center, 986025 Nebraska Medical Center, Omaha, NE 68198, \\ $U S A$
}

Received 5 August 2001; accepted 20 August 2001

\begin{abstract}
A new family of nanoscale materials on the basis of dispersed networks of cross-linked ionic and nonionic hydrophilic polymers is being developed. One example is the nanosized cationic network of cross-linked poly(ethylene oxide) (PEO) and polyethyleneimine (PEI), PEO-cl-PEI nanogel. Interaction of anionic amphiphilic molecules or oligonucleotides with PEO-cl-PEI results in formation of nanocomposite materials in which the hydrophobic regions from polyion-complexes are joined by the hydrophilic PEO chains. Formation of polyion-complexes leads to the collapse of the dispersed gel particles. However, the complexes form stable aqueous dispersions due to the stabilizing effect of the PEO chain. These systems allow for immobilization of negatively charged biologically active compounds such as retinoic acid, indomethacin and oligonucleotides (bound to polycation chains) or hydrophobic molecules (incorporated into nonpolar regions of polyionsurfactant complexes). The nanogel particles carrying biological active compounds have been modified with polypeptide ligands to enhance receptor-mediated delivery. Efficient cellular uptake and intracellular release of oligonucleotides immobilized in PEO-cl-PEI nanogel have been demonstrated. Antisense activity of an oligonucleotide in a cell model was elevated as a result of formulation of oligonucleotide with the nanogel. This delivery system has a potential of enhancing oral and brain bioavailability of oligonucleotides as demonstrated using polarized epithelial and brain microvessel endothelial cell monolayers. (C) 2002 Elsevier Science B.V. All rights reserved.
\end{abstract}

Keywords: Drug delivery; Drug targeting; Formulation; Nanogel; Polynucleotides; Polyethyleneimine; Poly(ethylene glycol)

\section{Contents}

1. Introduction

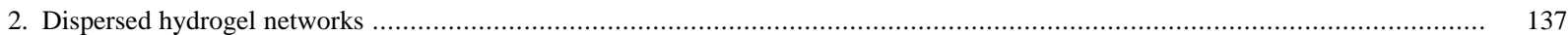

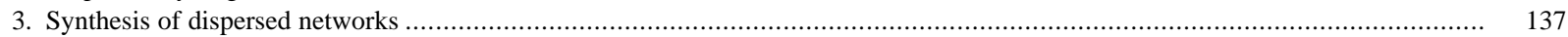

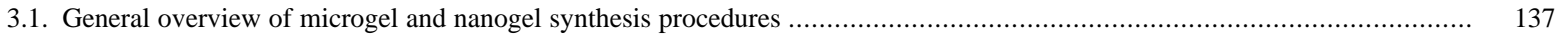

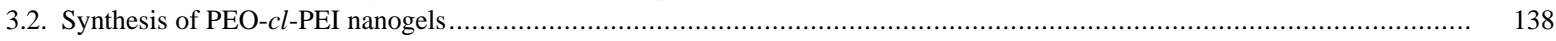

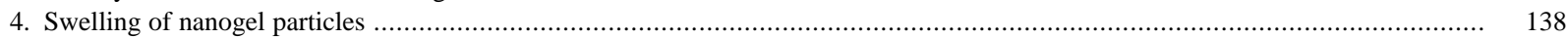

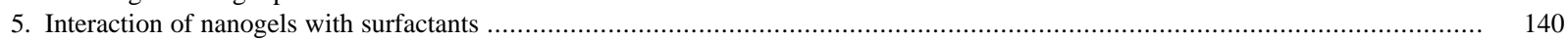

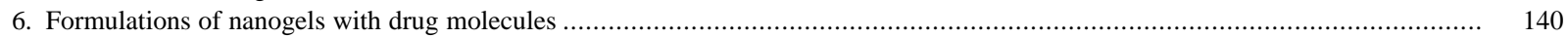




\section{Introduction}

Polymeric drug carriers of nanoscale size range have attracted increasing attention in recent years [1-5]. Polymers of synthetic and natural origin were used for preparation of such drug delivery systems. For example, biodegradable polyester nanoparticles are well-known carriers for low molecular mass drugs, oligonucleotides and peptides [2]. The advantages of using nanoparticles for drug delivery result from their two basic properties. First, nanoparticles, due to their small size, penetrate within even small capillaries and are taken up within cells, which allows for efficient drug accumulation at the target sites in the body [6-8]. Second, the use of biodegradable materials for nanoparticle preparation allows for sustained drug release within the target site over a period of days or even weeks after injection [9]. These advantages come at the substantial cost of a relatively low drug loading capacity and relatively complicated preparation procedure, which requires presence of the drug in the reaction mixture and often leads to the drug inactivation.

An alternative approach is to use swollen hydrophilic polymer gels of nanoscale size that can be synthesized in the absence of the drug and then loaded with the drug resulting in a gel collapse and formation of nanoparticles [10-12]. Drug loading is usually achieved through self-assembly mechanisms involving noncovalent interactions between the drug and the polymer matrix, such as electrostatic interactions, hydrogen bond formation and hydrophobic interactions. Additionally, drug molecules can be immobilized in the polymer gels via formation of a cleavable covalent bond between the drug moiety and the polymer matrix. The approach using dis- persed hydrogels is simple, results in relatively high drug loading capacity and may provide advantage from regulatory prospective because polymer gels are synthesized and evaluated in the absence of the drug. This paper overviews the preparation and use of nanoscale hydrogels for drug delivery. For simplicity these systems will be called 'the nanogels'. Along with this term we will be using the term 'microgels' to define gel particles of the micron scale size range.

Many of the examples of the pharmaceutical use of dispersed hydrogels that are discussed in this paper were obtained using nanogels from crosslinked poly(ethylene oxide) (PEO) and polyethyleneimine (PEI). This system represents a special type of a dispersed copolymer network in which cationic chains (PEI) alternate with hydrophilic nonionic chains (PEO). As a result of the double functionality, this system, called PEO-cl-PEI nanogel, exhibits combined properties of a swollen polyelectrolyte network and a hydrophilic nonionic network. Polyelectrolyte chains exhibit the ability to bind oppositely charged counterions or macromolecules, leading to the collapse of the gel, while, hydrophilic nonionic chains prevent precipitation and stabilize the particles in aqueous dispersions. PEO$c l$-PEI nanogels were reacted with a variety of materials including anionic surfactants and polynucleotides. Furthermore, substantial amount of data is now available regarding interaction of these materials with cells. This justifies the primary focus on the PEO-cl-PEI nanogels in the present review. Some other examples of dispersed networks from alternat-

${ }^{1}$ Nanogel $^{\mathrm{TM}}$ is a registered trademark of Supratek Pharma Inc. (Montreal, Canada). 
ing ionic and nonionic chains, particularly, poly(methacrylic acid) and PEO, can be also found in literature $[13,14]$.

\section{Dispersed hydrogel networks}

A gel is a three-dimensional cross-linked polymer network immersed in a fluid. Dispersed gel particles, microgels and nanogels, can be viewed as crosslinked latex particles, which are swollen by a good solvent. If the good solvent is water these species belong to a hydrogel class. Colloidal stability of such systems results primarily from matching of the Hamaker constants of the swollen particles and the solvent. These systems are currently being actively investigated due to their potential technological applications in a large number of areas: medicine, industry, and environmental cleanup. Applications of such materials include surface coating [15-17], uptake and release of heavy metal ions [18,19], drug delivery [20,21], optoelectronic switches [22], and others. The research in these fields is propelled by the interest in the ability of hydrogel materials to respond reversibly to external stimuli, such as temperature, $\mathrm{pH}$, ionic strength, solvent nature, and external stress. The microgel and nanogel particles are of particular interest because they exhibit intrinsic properties of gels combined with the properties of colloids, such as microheterogeneous structure, small size and high surface to volume ratio.

\section{Synthesis of dispersed networks}

\subsection{General overview of microgel and nanogel synthesis procedures}

Various approaches can be used for preparation of both bulk (macrogels) and dispersed gels. Macrogels in general are prepared using quiescent solutions in the presence of cross-linker and an accelerator. The products tend to be homogeneous with respect to composition. Microgel particles are usually synthesized by emulsion polymerization or copolymerization at elevated temperature using rapidly stirred solutions in order to obtain stable dispersions [23]. In this case the progression of the polymerization process towards macrogelation is constrained by the maximum size of the emulsion droplets. Structure and properties of microgels depend on composition and concentration of the monomers at the reaction sites, reactivity ratios, and solubility of the co-monomers in water. Diffusion control of the copolymerization rate is favored by (i) cross-linking, (ii) high concentration of the oil-soluble initiator and of the emulsifier, and (iii) low solubility of the comonomer in water [24]. One of the reactive components in the emulsion copolymerization can be used as an emulsifier. For example, the microgel particles of unsaturated polyester and styrene were prepared by cross-linking self-emulsifying copolymerization using unsaturated polyester with terminal carboxylic acid groups (1,6-hexanediol-maleic anhydride copolymer). In this process the polyester served as both the reactive component and emulsifier for the styrene monomer. It was shown that the size of the gel particles obtained by this method varied from $20 \mathrm{~nm}$ to $300 \mathrm{~nm}$ and strongly depended on the ratio between the components [25,26]. Microgels can be synthesized also by surfactant-free emulsion copolymerization, employing sonication instead of a conventional stirring technique [27]. By adjusting the ionic strength, initiator concentration and polymerization temperature, monodisperse polystyrene latexes with particle sizes $0.1-1.0 \mu \mathrm{m}$ were prepared by single-stage radical polymerization in the absence of surfactants [28,29]. In this case the total initial ionic strength determined the particle size in the latexes. Monodisperse, cationic microgels from 2-vinylpyridine [30] or from the copolymer of 2-vinylpyridine and styrene were synthesized using a cationic initiator 2,2'-azobis(2-amidinopropane) dihydrochloride [31]. Various anionic microgel particles were prepared by polymerizing a monomer mixture containing carboxylic acid monomers and vinyl monomers [16,18,32-34]. It was recently shown that the microgels and nanogels can be obtained by addition or step-growth polymerization of the polyfunctional monomers in solution [35]. A wide range of cross-linking systems and solvents were evaluated using this approach. To avoid macrogelation the polyfunctional precursors should be used below their critical gelation concentration, and the solvent solubility parameters should be carefully chosen for each polymer type made. It was hypoth- 
esized that the gels prepared using this technique contain (i) 'dead' chains, (ii) polymerizing chains, and (iii) chain loops, which all can act as effective steric stabilizers in a good solvent. These chains prevent particle-particle cross-linking and macrogelation resulting in formation of gel dispersion.

Polymeric gels containing several distinct types of chain fragments can be synthesized by polymerization of the monomer that forms one of the network fragments in the presence of another fragment(s). For example, polymerizing epoxides with polyalkylenepolyamines produced polymer beads [36]. Cationic microgel dispersions were produced by cross-linking polyepoxide-amine reaction products with polyepoxide cross-linking agent [15]. The nanosized poly(methacrylic acid-g-ethylene glycol) gels were prepared by micellar polymerization of methacrylic acid in the presence of poly(ethylene glycol) monomethyl ether monomethacrylate [37]. Another approach to synthesizing polymer networks involves cross-linking of pre-formed polymer fragments. For example, cross-linked polymer gels or films were prepared by cross-linking homopolymers and copolymers of acrylamide, N-substituted acrylamide or $\mathrm{N}$-substituted methacrylamide with polyamines or polyols [38]. The microgel particles were obtained by chemically cross-linking of the collapsed hydroxypropyl cellulose polymer chains in watersurfactant dispersions [39]. Overall, there are numerous methods of preparation of the microgel particles, which yield the dispersed networks with the desired characteristics. By varying the conditions of reaction, including the type and dispersity of the media, addition of surfactants, temperature, and ratio of reagents the size of the particles can be controlled. Many of the examples of the pharmaceutical use of dispersed hydrogels that are discussed in this paper were obtained using PEO-cl-PEI nanogels. The synthesis of these materials is considered in greater detail in the next section.

\subsection{Synthesis of PEO-cl-PEI nanogels}

PEO- $c l$-PEI nanogels were synthesized by crosslinking of branched PEI ( $25 \mathrm{kDa})$ with bis-activated PEO (8 kDa) molecules [10]. When conducted in a homogeneous aqueous solution the reaction between amino groups of PEI and imidazolylcarbonyl ends of activated PEO proceeded very rapidly resulting in formation of transparent hydrogels in only 3-5 min. These bulk hydrogels retained large quantities of water reaching approximately 50 -fold by weight compared to the dried substance. Rigid hydrogels could be produced at the minimal PEO/PEI molar ratio 6 or higher. To obtain fine dispersed systems, the cross-linking reaction was performed by a modified solvent emulsification/evaporation method [40]. According to this method, activated PEO solution in dichloromethane was emulsified in the aqueous solution of PEI by sonication (Fig. 1). The organic solvent was removed from the mixture in vacuo resulting in formation of a clear suspension. This suspension was centrifuged to remove debris and large particles. Most of the nanogel particles had a very low density and could not be fractionated by ultracentrifugation. Therefore, crude suspension of nanogel particles was purified using gel-permeation chromatography. Several fractions could be separated by particle size from 300 to $40 \mathrm{~nm}$, with a major fraction (ca. $70 \%$ of the product) having average particle diameter between 150 and $240 \mathrm{~nm}$. The quantity of PEO and PEI components in these nanogel preparations was determined from ${ }^{1} \mathrm{H}-\mathrm{NMR}$ spectra the average PEO/PEI ratios ranged from 6 to 7.

\section{Swelling of nanogel particles}

The aqueous dispersions of the PEO-cl-PEI particles were prepared by dissolving of lyophilized powder of the copolymer in the deionized water or buffer solution followed by bath-sonication for 3-4 min. It is well recognized that a balance between the osmotic pressure and the polymer elasticity sets the physical dimensions of a hydrogel microsphere [41]. The osmotic pressure results from the net difference in concentration of mobile ions between the interior of the microgel particle and exterior solution. For ionic polymer gels fixed charged groups attract hydrated counterions, which tend to expand the gel, while the conformational entropy elasticity of the cross-linked polymer chains opposes this expansion. Neutralizing the gel reduces the net ion concentration difference (osmotic swelling pressure). This reduction in the number of counterions results in dehydra- 

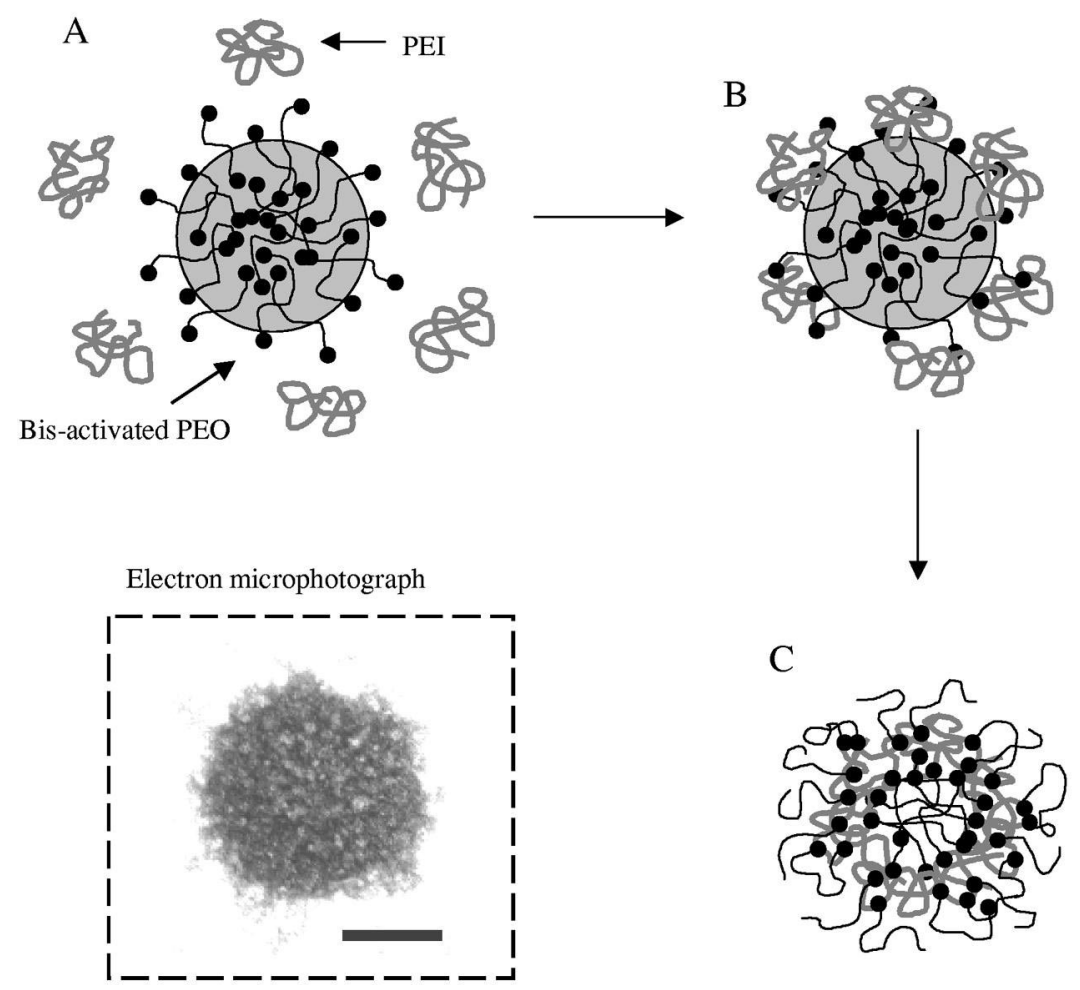

Fig. 1. Schematic representation of the synthesis of the PEO-cl-PEI nanogel using emulsification-evaporation method: (A) droplets of dichloromethane (grey color) containing bis-activated PEO (both ends activated by $1,1^{\prime}$-carbonyldiimidazole) is dispersed in the bulk aqueous solution cantaining PEI; (B) reaction between PEO and PEI proceeds on the surface of the o/w emulsion droplets; (C) organic solvent is evaporated and the nanogel is maturated for $24 \mathrm{~h}$ in aqueous dispersion. The insert presents transmission electron micrograph of mature nanogel particle (staining with uranyl acetate, $180000 \times$, bar $100 \mathrm{~nm}$ ).

tion of the gel and decrease in the volume to an extent when the excluded volume of the polymer chains limits the further compression. The polyamine segments of PEO-cl-PEI nanogels are weak polybases and the charge density of such networks depends on $\mathrm{pH}$. Furthermore, PEI segments are randomly branched, i.e. contain primary, secondary and tertiary amino groups. As it was estimated by potentiometric titration only approximately $60 \%$ of the total amount of amino groups could be protonated in the range of $\mathrm{pH}$ from 10 to 4 [42]. Despite of the relatively high concentration of fixed charged groups the PEO-cl-PEI nanogels exhibited relatively little size variation upon changes in $\mathrm{pH}$. At $\mathrm{pH} 10$ when practically all amino groups of PEI are uncharged the nanogel particle size was ca. $260 \mathrm{~nm}$. The swelling of PEO-cl-PEI particles under these conditions is mainly due to the effects of the excluded volumes of hydrophilic PEI and PEO chains. The protonation of amino groups upon lowering of $\mathrm{pH}$ to 8.5 resulted in the swelling of PEO-cl-PEI particles and increase in the size of the particles up to ca. $300 \mathrm{~nm}$. However, the particle size remained practically constant upon further decrease of $\mathrm{pH}$ in the range from 8.5 to 4.0. This behavior was attributed to a relatively high degree of crosslinking of the PEO-cl-PEI network (ca. 1 PEO chain per 80 units of PEI) that limits the extent of its swelling. From the experimental perspective one of the advantages to work with gels in nanosize and submicron range is the fact that they undergo a very rapid response to changing environmental conditions $[33,34]$. In contrast, the swelling equilibrium for the macrogels requires periods of the order of days [43]. 


\section{Interaction of nanogels with surfactants}

Interaction between polyions and oppositely charged surfactants is a cooperative process in which the ionic head-groups of the surfactant bind to the polyion repeating units while the surfactant alkyl groups segregate into hydrophobic domains [44]. Binding of various anionic surfactants with cationic polyamine segments of PEO-cl-PEI networks was studied by fluorescence probe (pyrene) technique [45]. This process characterized by a 'critical association concentration' (cac), indicating the onset of surfactant binding to the polyion chains of network. For instance, for sodium tetradecyl sulfate the cac value in the presence of PEO-cl-PEI was $3.2 \times 10^{-5}$ $\mathrm{M}$ and was about 2 order of magnitude lower than the corresponding $\mathrm{cmc}\left(2.2 \times 10^{-3} \mathrm{M}\right)$, suggesting that PEO-cl-PEI copolymer promoted the surfactant aggregation. The shift in cac to lower concentrations compared to the cmc is explained by the cooperative stabilization of surfactant aggregates as a result of interaction between surfactant and PEI chains of the network.

The complexes of PEO-cl-PEI with surfactants were characterized by laser microelectrophoresis technique and photon correlation spectroscopy at various compositions of the mixture [45]. The composition of the mixture, $N / S$, is expressed as a ratio of the total concentration of amino groups of PEO$c l$-PEI to the concentration of the surfactant in the system. In all experiments the concentration of amino groups (N) was constant while the concentration of the surfactant was varied. Since only a fraction of amino groups could be protonated at $\mathrm{pH}$ 7.0 [42], the stoichiometric compositions of the mixtures at this $\mathrm{pH}$ corresponded to $\mathrm{N} / \mathrm{S} \approx 2.5$. The net charge of the unloaded PEO-cl-PEI particles was slightly positive. The small values of the zeta-potential of these particles are obviously a result of localization of a significant portion of the charged amino groups and the condensed counterions within the network particles. This should lead to a decrease in the electric potential at the surface of shear of such particles. Incorporation of surfactant molecules into PEO-cl-PEI resulted in a decrease of zeta-potential almost to zero. When an excess of surfactant was added to the system $(N / S<2.5)$ the net charge of the particles became slightly negative. The change in the sign of zeta-potential can be attributed to incorporation of the excess of the surfactant into the PEO-cl-PEI/surfactant complexes. Surfactant molecules can bind with the stoichiometric complexes through interactions with the hydrophobic domains and/or PEO segments of the network.

The binding of anionic surfactants to the amino groups of ionic segments of PEO-cl-PEI networks led to a decrease in the particles size to about $50 \mathrm{~nm}$ (compare with the initial size of $300 \mathrm{~nm}$ ) [45]. A sharp decrease in the size of the particles was observed even under the conditions of the deficiency of the surfactant in the reaction mixture $(N / S>2.5)$. The contraction of PEO-cl-PEI networks as a result of surfactant binding was also confirmed by electron microscopy. Electron micrographs revealed close to spherical PEO-cl-PEI particles, which collapsed upon the interaction with amphiphilic molecules. Based upon the changes of diameters of the particles before and after addition of surfactants, as revealed by both light scattering and electron microscopy, the volume of the particles decreased more than 100-fold upon interaction of the network with surfactants. A network collapse is typical for polyelectrolyte gels upon their interaction with oppositely charged surfactants [46]. It is noteworthy, however, that despite charge neutralization PEO- $\mathrm{cl}$-PEI/surfactant complexes formed fine, slightly opalescent dispersions, which exhibited no precipitation in the entire ranges of $N / S$ and $\mathrm{pH}$ examined.

\section{Formulations of nanogels with drug molecules}

Many biologically active compounds are characterized by relatively low solubility, which hinders their pharmacological use. At the same time some of these compounds are ionogenic surfactants that can be used as components of block ionomer complexes for drug delivery [47]. Incorporation of amphiphilic molecules with low solubility into PEO-cl-PEI nanogels was evaluated using sodium oleate (OA), indomethacin, and retinoic acid (RA) [45]. In the case of $\mathrm{OA}$ and indomethacin the complexes were prepared by mixing small amounts of concentrated solutions of the surfactants in organic solvent (etha- 
nol, dimethylformamide) with aqueous dispersions of PEO-cl-PEI ( $\mathrm{pH}$ 7.0). For both studied compounds the fine dispersions of the complex particles were obtained in the wide range of compositions of the mixture. These formulations were stable over relatively long periods of time (at least a week). However, this protocol was not successful in the case of RA. Mixing of PEO-cl-PEI and RA at $\mathrm{pH} 7.0$ resulted in immediate precipitation. Therefore, the complexes were prepared by mixing of the components at $\mathrm{pH} 10$, under the conditions when RA is completely ionized and stable (N/S ratio 3.0). This procedure produced a practically transparent and stable dispersion with the particle size of ca. $70 \mathrm{~nm}$. After formation at $\mathrm{pH} 10.0$ the $\mathrm{pH}$ of dispersion was adjusted to 7.0. The $\mathrm{pH}$ adjustment did not result in a change of the size of the particles. Furthermore, the dispersion remained stable in $0.15 \mathrm{M} \mathrm{NaCl}$. In the absence of added salt this dispersion could be lyophilized, stored in lyophilized form for several days, and then re-dispersed. This suggested that a useful pharmaceutical formulation of RA can be prepared by its immobilization in the nanogel system, which is stable at physiological $\mathrm{pH}$ and ionic strength [45].

The kinetic of drug release from dispersion of nanogel loaded with indomethacin was evaluated using the equilibrium dialysis technique [45]. This experiment suggested that during the first hour of dialysis over $17.5 \%$ of the drug was released in the external solution. After $24 \mathrm{~h}$ ca. $82 \%$ of indomethacin was found in the external solution. The release of the drug from PEO- $c$-PEI nanogels results in the restoration of the nanogel size. Indeed, the estimated effective diameter of the PEO-cl-PEI particles after dialysis was ca. $270 \mathrm{~nm}$. This demonstrates that the biological agents immobilized within the nanogel can be released from it and suggests that such systems may be useful for drug delivery applications.

The hydrophobic regions of PEO-cl-PEI/surfactant complexes can also serve as non-aqueous reservoirs for solubilizing water insoluble molecules. For example, PEO-cl-PEI/OA complexes incorporated a significant amount of water-insoluble dye, Yellow OB [45]. The dye-containing PEO-cl-PEI/OA dispersions remained stable for several days. These data suggested that PEO-cl-PEI/surfactant dispersions can potentially be used for solubilization of hydrophobic drugs.

\section{Formulations of PEO-cl-PEI nanogels with oligonucleotides}

Addition of oligonucleotide to PEO-cl-PEI nanogel dispersion at $\mathrm{pH} 7.0$ resulted in immediate formation of polyelectrolyte complexes due to the binding of oligonucleotide molecules to the PEI chain fragments of the nanogel [10]. Like in the case of surfactants discussed above, the properties on the resulting complexes depended on the ratio of the concentrations of the reacting oppositely charged species. In the case of the polynucleotide-based complexes the ratio of total nitrogen of PEO-cl-PEI to the phosphates of polynucleotide, called ' $N / P$ ratio' defines the composition of the mixture. The zeta-potential of the PEO-cl-PEI/oligonucleotide complexes was close to zero at $N / P$ ratio 4 . Gel permeation HPLC analysis of the oligonucleotide and PEO- $c l$-PEI mixtures showed no free oligonucleotide in solution at $N / P$ ratios 4 and higher, suggesting that all oligonucleotide molecules incorporated in the nanogel. The PEO-cl-PEI nanogel loading capacity with respect to the oligonucleotide was $150 \mu \mathrm{g} \mathrm{mg}^{-1}$ i.e. ca. $15 \%$ by weight. At lower $N / P$ ratios (i.e. higher oligonucleotide content) free oligonucleotide was observed in the mixture suggesting that the maximal loading capacity was achieved at or about $N / P$ ratio 4 . The particle size decreased upon adding the polynucleotide. In the vicinity of the neutralization point at $N / P$ ratio 4 , the minimum particle size of ca. $80 \mathrm{~nm}$. was observed. Nanogel particles revealed the tendency to slowly aggregate at the electroneutrality point. However, no aggregation was observed at $N / P$ ratio higher than 4 when particle exhibited small positive charge.

\section{Modification of the surface of nanogel particles with biospecific ligands}

To allow for the targeted delivery of nanogels in the body the surface of the nanogel particles can be modified with various biospecific ligands. Various coupling strategies can be used for this purpose 
including covalent attachment of the ligand moiety to the free amino groups of the PEI fragments in PEO-cl-PEI nanogel. One simple way to introduce various ligands in nanogel particles consists in the partial modification of PEI fragments with biotin moieties [10]. Biotin $p$-nitrophenyl ester was used for modification of amino groups of PEO-cl-PEI. The amount of biotin moieties conjugated to PEO- $c l$ PEI was determined using analytical assay based on the competitive displacement of a fluorescent dye, 2-anilinonaphthalene-6-sulfonic acid, in the reaction with avidin [48]. The biotinylated PEO-cl-PEI nanogel was further reacted with oligonucleotide resulting in the formation of the particles, which were very similar to the ones described in the previous section. However, the surface of these particles also contained the biotin groups, which can be used for attachment of various vector moieties (e.g., peptides and antibodies) using standard biotinavidin coupling chemistry. In the model experiments these particles were coupled with the biotinylated transferrin and biotinylated insulin through the avidin molecule [10]. The modification technique described in this chapter serves only as one example of possible strategy for targeting nanogels using biospecific ligands. Obviously, there could be other strategies developed for this purpose including direct conjugation of the ligand with the nanogel. It should be noted that direct conjugation of a ligand to a positively charged polymer backbone is associated with concerns regarding changes in the ligand specificity. Therefore, a prudent approach for vectorization of nanogels might include coupling of a ligand through a neutral polymer spacer such as coupling of ligand at the free ends of PEO chains linked to the nanogel surface.

\section{Effect of the particle size on the biological properties of drug carriers}

From the drug delivery standpoint, the size of the particles is particularly important. Size variation within the nanoscale range will strongly affect both the blood circulation time as well as the bioavailability of the particles within the body [49-54]. Typically, following systemic administration, small particles with diameters of less than 5-10 $\mathrm{nm}$ are rapidly removed through extravasation and renal clearance, while larger particles with diameters of more than $10 \mathrm{~nm}$ are not removed by these means [50]. Particles ranging from ca. 10 to $70 \mathrm{~nm}$ are still small enough to penetrate even the very small capillaries within the body tissues, and therefore may offer the most effective distribution in certain tissues $[49,51]$. For example, particles in this size range are optimal for subcutaneous injection since the particles are more rapidly drained from the injection site [51]. Slightly larger particles, ranging from ca. 70 to 200 $\mathrm{nm}$, demonstrate the most prolonged circulation times [49,53]. In contrast, even larger particles, with diameters greater than $200 \mathrm{~nm}$, are usually sequestered by the spleen as a result of mechanical filtration and are eventually removed by the cells of the phagocyte system [49,52], resulting in decreased blood circulation times. This information, combined with the knowledge that particles which are smaller than $100 \mathrm{~nm}$ can be enclosed within endocytic vesicles, allowing entry into target cells via endocytosis [55], leads to the conclusion that the preferred size range for drug delivery using nanoscale particles is from ca. 10 to $100 \mathrm{~nm}$. Therefore, the average hydrodynamic diameters observed for the loaded PEO-cl-PEI nanogel fit well within the preferred size range for drug delivery systems.

\section{Uptake of oligonucleotide-loaded nanogel within the cells}

Positively charged nanoscale particles are believed to bind electrostatically with the negatively charged cell membranes, which is followed by the internalization of these particles within the cells through the adsorptive endocytosis [56]. The study of the internalization of the oligonucleotide-loaded PEO-cl-PEI nanogel suggested that the positively charged particles $(N / P$ ratio 8$)$ are much more efficiently taken up within the cells than the electroneutral particles $(N / P$ ratio 4$)$ [10]. Therefore, to enhance the uptake of the loaded nanogel particles these particles were coupled with insulin and transferrin using the biotinavidin coupling technique. As a result a drastic enhancement of accumulation of the particles within the cells was observed.

Fluorescently tagged oligonucleotides were used 
to examine localization of the oligonucleotides within the cell. Following internalization by endocytosis, the major portion of free oligonucleotides is usually sequestered within endosomal and lysosomal compartments of the cells [57]. Less significant amounts of oligonucleotides are also distributed within other cellular organelles such as the Golgi complex and the Endoplasmic reticulum [57]. Therefore, addition of chloroquine is commonly required to enhance release of the oligonucleotide in the cytoplasm and delivery of the oligonucleotide to the nucleus of the cell [58]. In the case of the oligonucleotides delivered using the PEO-cl-PEI nanogel effective release of the oligonucleotide and its accumulation within the nucleus was observed in the absence of chloroquine [10]. It is possible that following the internalization of the PEO-cl-PEI/oligonucleotide complexes within the endosomes the cationic PEI chains interact electrostatically with negatively charged membrane phospholipids, which locally destabilizes the endosomal membrane. Furthermore such interaction should also facilitate the displacement and release of the oligonucleotide from the complex with the nanogel. It is noteworthy that following internalization of the PEO-cl-PEI/oligonucleotide complexes within the cells the nanogel particles are found in the cytoplasmic compartments of the cells, while the oligonucleotides accumulate predominantly in the nucleus (unpublished results). The schematic illustrating the hypothetical mechanism of the oligonucleotide delivery within the cell is presented in Fig. 2. Transport of oligonucleotide delivered with

A

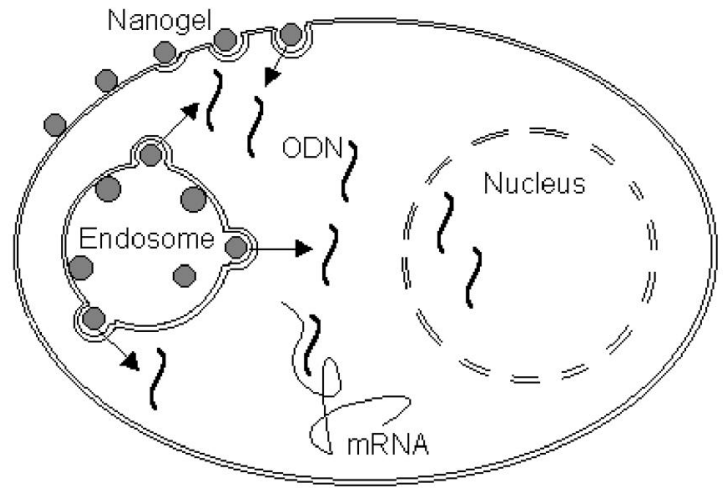

B

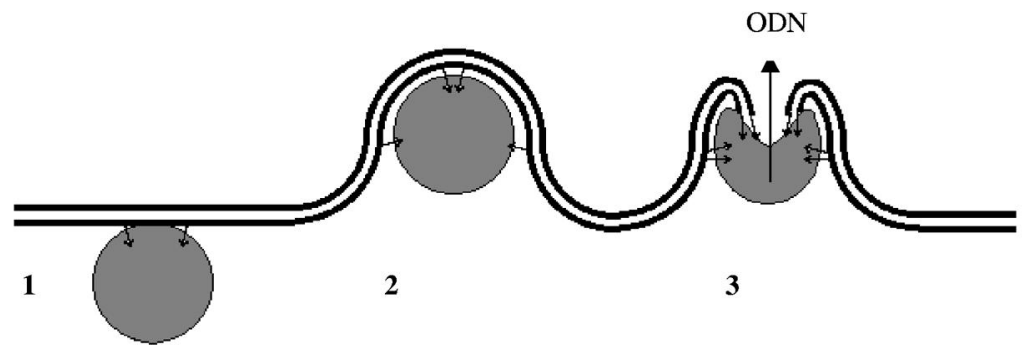

Fig. 2. (A) Internalization of nanogel/oligonucleotide (ODN) particles by adsorptive mediated endocytosis and putative mechanism of ODN unloading into the cytosol. (B) Destabilization of the endosomal membrane: (1) interaction of positively charged nanogel particles with negatively charged phospholipids; (2) nanogel wrapping with interacting phospholipids and (3) collapse of nanogel-phospholipid complex and relaese of SODN into the cytosol. 
nanogel within the nucleus of the cells is remarkable in view of the well-known difficulties of DNA delivery to the nucleus of nondividing cells. However, small size of oligonucleotide molecule compared to the size of the plasmid DNA might be beneficial for its nuclear delivery, since oligonucleotide can diffuse through the cytoplasm and penetrate the pores in the nuclear membrane. It appears that the oligonucleotide is released within the cell prior to delivery to the nucleus since relatively large oligonucleotide-nanogel complex cannot cross the nuclear membrane. Further studies of intracellular trafficking of molecules delivered with nanogels is currently in progress.

\section{Biological activity of antisense oligonucleotide delivered using nanogel}

Several recent reports have documented the advantages of polymer particles as effective in vitro and in vivo delivery systems for antisense oligonucleotides [2,59]. Using antisense oligonucleotide directed against mRNA of membrane P-glycoprotein (Pgp) the biological effect of oligonucleotides immobilized in the PEO- $c l$-PEI particles was evaluated in several multidrug resistant cell lines [10]. This study demonstrated significant enhancement of antisense inhibition of Pgp as a result of incorporation of oligonucleotide in the nanogel as determined using both Pgp functional assay and the polymerase chain reaction. Furthermore, the antisense effects were significantly enhanced when the PEO-cl-PEI/oligonucleotide complexes were modified with transferrin using the avidin-biotin coupling technique.

\section{Delivery of oligonucleotides across gastrointestinal epithelium}

Oral administration of oligonucleotides would be advantageous since it offers greater patient compliance. However, oligonucleotides are rapidly degraded in the gastrointestinal tract and their bioavailability is less than $1 \%$ [2]. Poor oral bioavailability of oligonucleotides is to a significant extent due to the low efficacy of their transport across gastrointestinal epithelium [60]. In order to evaluate the possibility of using PEO-cl-PEI nanogel for oral administration of oligonucleotides, the permeability studies were carried out using polarized Caco- 2 cell monolayers and standard Side-Bi-Side two-chamber device as an in vitro model of gastrointestinal epithelium [10]. The transfer of the free or nanogelincorporated oligonucleotide in the receiver chamber was assayed using a fluorescence technique. As is seen in Fig. 3B, incorporation of the oligonucleotide in nanogel results in a drastic increase in the oligonucleotide transport across the Caco-2 cell monolayers compared to the free oligonucleotide transport. The transfer of a paracellular marker, ${ }^{3} \mathrm{H}$ mannitol, in the presence of PEO-cl-PEI/oligonu-
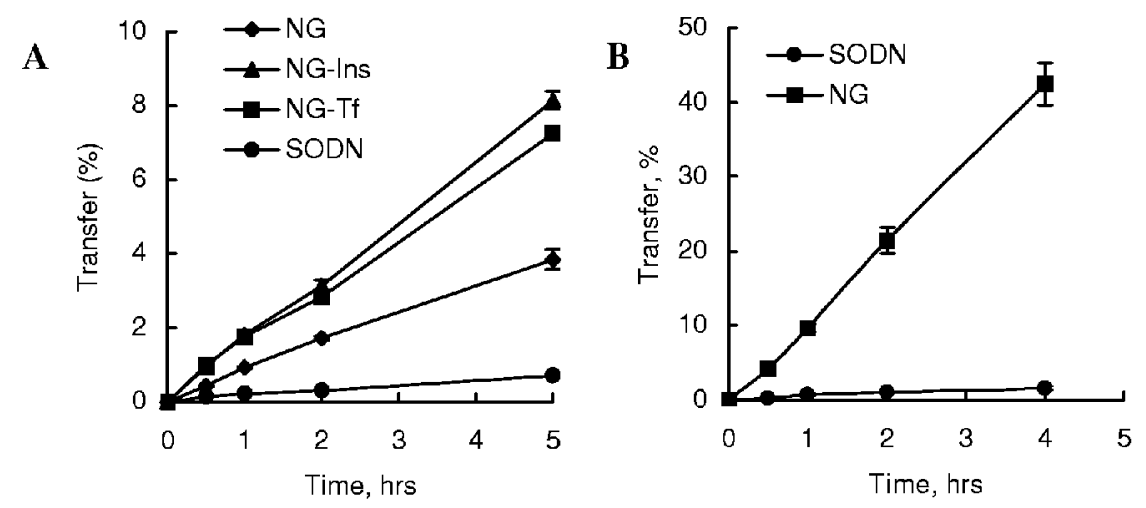

Fig. 3. Permeability of SODN and SODN-loaded nanogel carriers in BBMEC (A) and Caco-2 (B) cell manolayers. SODN concentration in all experiments was $5 \mu \mathrm{M}$. Nanogel particles were loaded at $N / P$ ratio $4(\mathrm{~A})$ and $8(\mathrm{~B})$. Concentration of human transferrin and insulin in the system was $2.5 \mu \mathrm{M}$. Quantity of the SODN passed through the cell monolayers was measured by analytical anion exchange HPLC. 
cleotide complexes was not affected, suggesting that the observed enhancement of the oligonucleotide permeability in Caco-2 cell monolayers is due to the enhancement in the transcellular delivery route rather increased leakiness of the cell monolayers. Finally, the analysis of the oligonucleotide in the receiver chamber using HPLC demonstrated that the oligonucleotide immobilized in nanogel particles crossed intestinal cell monolayers without degradation. As a result of this work, it appears likely that one useful application of the nanogel carriers may be in oral administration of antisense oligonucleotides.

\section{Delivery of oligonucleotides across blood- brain barrier}

Brain has recently attracted significant attention as a potential target tissue for drug delivery. The sequestered nature of the central nervous system makes delivery of drugs to the brain a challenging task. At present there are few reports of successful delivery of drugs across the blood-brain barrier (BBB), which involve the use of the block copolymers, polymer micelles, liposomes and nanoparticles [61-63]. In this respect it was of interest to evaluate the potential of using nanogel carriers for drug delivery to the brain. The studies examined delivery of oligonucleotides across the BBB using polarized monolayers of bovine brain microvessel endothelial cells (BBMEC) as an in vitro model of the $\mathrm{BBB}$. The experiments were conducted similarly to those described in the previous section. As is shown in Fig. 3A the transport of oligonucleotide across the cell monolayers was significantly increased as a result of incorporation of oligonucleotide in the PEO-cl-PEI nanogel. Further increase in the oligonucleotide transport was observed when the nanogel carriers were modified with insulin or transferrin vectors, which both were shown to be transported across $\mathrm{BBB}$ through a receptormediated transcytosis mechanism $[64,65]$. While the improved brain bioavailability of oligonucleotides using nanogels needs to be further validated in in vivo studies, the current result is encouraging in view of the low efficiency of oligonucleotide delivery across brain microvessel endothelial cells. Overall, it suggests that nanogels have a potential to enhance current strategies for vector-mediated drug delivery to the brain [66].

\section{Conclusions}

In conclusion, nanogels exemplified in this paper using dispersed PEO-cl-PEI networks, appear to be promising and versatile systems for drug delivery. The advantages of these systems include simplicity of formulation with the drugs, high loading capacity and stability of the resulting formulation in dispersion. These systems allow immobilization of biologically active compounds of diverse structure including charged drugs, low molecular mass hydrophobes and biopolymers. In addition to the data presented in this chapter in more recent studies nanogels were used for immobilization of plasmid DNA and small globular proteins, which will be reported separately. Furthermore, nanogels can be chemically modified to incorporate various ligands for targeted drug delivery. The in vitro studies suggest that nanogels can be used for efficient delivery of biopharmaceuticals in cells as well as for increasing drug delivery across cellular barriers. The in vivo evaluation of nanogels is underway.

\section{Acknowledgements}

The authors are grateful for the support of this work by NSF (BES-9986393, DMR-0071682). Supratek Pharma Inc (Canada) supported synthesis of PEO-cl-PEI. AVK serves as a consultant to this company.

\section{References}

[1] J. Kreuter, Nanoparticles and nanocapsules - new dosage forms in the nanometer size range, Pharm. Acta Helv. 53 (1978) 33-39.

[2] G. Lambert, E. Fattal, P. Couvreur, Nanoparticulate systems for the delivery of antisense oligonucleotides, Adv. Drug. Deliv. Rev. 47 (2001) 99-112.

[3] S.M. Moghimi, L. Illum, S.S. Davis, Physiopathological and physicochemical considerations in targeting of colloids and drug carriers to the bone marrow, Crit. Rev. Ther. Drug. Carrier Syst. 7 (1990) 187-209. 
[4] G.J. Russell-Jones, Oral vaccine delivery, J. Control Release 65 (2000) 49-54.

[5] V.P. Torchilin, Polymer-coated long-circulating microparticulate pharmaceuticals, J. Microencapsul. 15 (1998) 1-19.

[6] Discussion S183-S184 O. Clement, F. Rety, C.A. Cuenod, N. Siauve, F. Carnot, C. Bordat, M. Siche, G. Frija, MR lymphography: evidence of extravasation of superparamagnetic nanoparticles into the lymph, Suppl 1 Acad. Radiol. 5 (1998) S170-S172.

[7] W.L. Monsky, D. Fukumura, T. Gohongi, M. Ancukiewcz, H.A. Weich, V.P. Torchilin, F. Yuan, R.K. Jain, Augmentation of transvascular transport of macromolecules and nanoparticles in tumors using vascular endothelial growth factor, Cancer Res. 59 (1999) 4129-4135.

[8] H. Winet, J.O. Hollinger, M. Stevanovic, Incorporation of polylactide-polyglycolide in a cortical defect: neoangiogenesis and blood supply in a bone chamber, J. Orthop. Res. 13 (1995) 679-689.

[9] L.A. Guzman, V. Labhasetwar, C. Song, Y. Jang, A.M. Lincoff, R. Levy, E.J. Topol, Local intraluminal infusion of biodegradable polymeric nanoparticles. A novel approach for prolonged drug delivery after balloon angioplasty, Circulation 94 (1996) 1441-1448.

[10] S. Vinogradov, E. Batrakova, A. Kabanov, Poly(ethylene glycol)-polyethylenimine NanoGel particles: novel drug delivery systems for antisense oligonucleotides, Colloids Surf., B 16 (1999) 291-304.

[11] P. Lemieux, S.V. Vinogradov, C.L. Gebhart, N. Guerin, G. Paradis, H.K. Nguyen, B. Ochietti, Y.G. Suzdaltseva, E.V. Bartakova, T.K. Bronich, Y. St-Pierre, V.Y. Alakhov, A.V. Kabanov, Block and graft copolymers and NanoGel copolymer networks for DNA delivery into cell, J. Drug Target. 8 (2000) 91-105.

[12] Y. Shin, J.H. Chang, J. Liu, R. Williford, G.J. Exarhos, Hybrid nanogels for sustainable positive thermosensitive drug release, J. Control Release 73 (2001) 1-6.

[13] A.M. Lowman, N.A. Peppas, Molecular analysis of interpolymer complexation in graft copolymer networks, Polymer 41 (1999) 73-80.

[14] P. Bures, N.A. Peppas, A new dispersion technique for micro- and nanoparticle formation of drug delivery carriers based on poly(acrylic acid), in: Proc. Int. Symp. Controlled Release Bioact. Mater, 27th, 2000, pp. 1102-1103.

[15] V.G. Corrigan, S.R. Zawacky, Cationic microgels and their use in electrodeposition US5,096,556, 1992.

[16] C. Gajria, Y. Ozari, Water-swellable crosslinked polymeric microgel particles and aqueous dispersions of organic filmforming resins containing the same, in: US 4,560,714, 1985.

[17] O. Quadrat, J. Snuparek, Structure and flow properties of latexes containing carboxylic groups, Prog. Org. Coat. 18 (1990) 207-228.

[18] A. Biffis, Functionalised microgels: novel stabilizers for catalytically active metal colloids, J. Mol. Catal. A: Chem. 165 (2001) 303-307.

[19] G.E. Morris, B. Vincent, M.J. Snowden, The interaction of thermosensitive, anionic microgels with metal ion solution species, Prog. Colloid Polym. Sci. 105 (1997) 16-22.
[20] N.A. Peppas, P. Bures, W. Leobandung, H. Ichikawa, Hydrogels in pharmaceutical formulations, Eur. J. Pharm. Biopharm. 50 (2000) 27-46.

[21] P.F. Kiserab, G. Wilson, D. Needham, Lipid-coated microgels for the triggered release of doxorubicin, J. Control Release 68 (2000) 9-22.

[22] T. Sawai, Y. Ikariyama, M. Aizawa, Electrically modulated/ flocculation of ultra-fine microgels, in: Proc. Int. Conf. Intell. Mater, 1993, pp. 279-282.

[23] M.J. Murray, M.J. Snowden, The preparation, characteriztion and applications of colloidal microgels, Adv. Colloid Interface Sci. 54 (1995) 73-91.

[24] J. Kaczun, W. Funke, Molecular design of reactive microgels, Angew. Makromol. Chem. 240 (1996) 99-112.

[25] L. Liang, W. Funke, Cross-linking self-emulsifying copolymerization of an unsaturated polyester and styrene, Macromolecules 29 (1996) 8650-8655.

[26] Y.C. Yu, W. Funke, Reactive microgels by emulsion polymerization of unsaturated polyester resins, Angew. Makromol. Chem. 103 (1982) 187-202.

[27] K. Kratz, T. Hellweg, W. Eimer, Influence of charge density on the swelling of colloidal poly( $\mathrm{N}$-isopropylacrylamide-coacrylic acid) microgels, Colloids Surf., A 170 (2000) 137149.

[28] J.W. Goodwin, J. Hearn, C.C. Ho, R.H. Ottewill, Preparation and characterization of monodisperse polystyrene latexes III. Preparation without added surface active agents, Colloid Polym. Sci. 252 (1974) 464-471.

[29] J.W. Goodwin, J. Hearn, C.C. Ho, R.H. Ottewill, Preparation and characterization of polymer latices formed in the absence of surface active agents, Brit. Polym. J. 5 (1973) 347-362.

[30] A. Fernandez-Nieves, A. Fernandez-Barbero, B. Vincent, F.J. de las Nieves, Charge controlled swelling of microgel particles, Macromolecules 33 (2000) 2114-2118.

[31] A. Loxley, B. Vincent, Equilibrium and kinetic aspects of the $\mathrm{pH}$-dependent swelling of poly(2-vinylpyridine-co-styrene) microgels, Colloid Polym. Sci. 275 (1997) 1108-1114.

[32] G.M. Eihebaum, P.F. Kiser, D. Shah, S.A. Simon, D. Needman, Investigation of the swelling response and drug loading of ionic microgels: the dependence on functional group compositon, Macromolecules 32 (1999) 8996-9006.

[33] G.M. Eihebaum, P.F. Kiser, S.A. Simon, D. Needman, pH and ion-triggered volume response of anionic hydrogel microspheres, Macromolecules 31 (1998) 5084-5093.

[34] B.R. Saunders, H.M. Crowther, B. Vincent, Poly[(methyl methacrylate)-co-(methacrylic acid)] microgel particles: swelling control using $\mathrm{pH}$, cononsolvency, and osmotic deswelling, Macromolecules 30 (1997) 482-487.

[35] N.B. Graham, A. Cameron, Nanogels and microgels: the new polymeric materials playground, Pure Appl. Chem. 70 (1998) 1271-1275.

[36] E.L. Ward, Reaction product of an epoxide and a polyalkylenepolyamine in bead form and a method for preparing same, in: US 4,189,539, 1980.

[37] H. Ichikawa, N.A. Peppas, pH-dependent swelling of nanosized poly(methacrylic acid-g-ethylene glycol) gels, Polymer Prep. 40 (1999) 363-364. 
[38] H.P. Gregor, E. Samuelson, P.I. Dalven, C.D. Gregor, Homopolymers and copolymers of acrylamide N-substituted acrylamide or $\mathrm{N}$-substituted methacrylamide solid state cross-linked with polyamines or polyols, in: US 5,280,078, 1994.

[39] X. Lu, Z. Hu, J. Gao, Synthesis and light scattering study of hydroxypropyl cellulose microgels, Macromolecules 33 (2000) 8698-8702.

[40] P.J. Watts, M.C. Davies, C.D. Melia, Microencapsulation using emulsification/solvent evaporation: an overview of techniques and applications, Crit. Rev. Ther. Drug Carrier Syst. 7 (1990) 235-259.

[41] J. Ricka, T. Tanaka, Swelling of ionic gels: quantitative performance of the Donnan theory, Macromolecules 17 (1984) 2916-2921.

[42] S.V. Vinogradov, T.K. Bronich, A.V. Kabanov, Self-assembly of polyamine-poly(ethylene glycol) copolymers with phosphorothioate oligonucleotides, Bioconjug. Chem. 9 (1998) 805-812.

[43] A. Katchalsky, Polyelectrolyte gels, Prog. Biophys. 4 (1954) $1-59$.

[44] E.D. Goddard, K.P. Ananthapadmanabhan (Eds.), Interactions of Surfactants With Polymers and Proteins, CRC Press, Boca Raton, FL, 1993.

[45] T.K. Bronich, S.V. Vinogradov, A.V. Kabanov, Interaction of nanosized copolymer networks with oppositely charged amphiphilic molecules, Nano Lett. 1 (2001) 535-540.

[46] V.A. Kabanov, A.B. Zezin, V.B. Rogacheva, Y.V. Khandurina, O.A. Novoskoltseva, Absorption of ionic amphiphiles by oppositely charged polyelectrolyte gels, Macromol. Symp. 126 (1997) 79-94.

[47] T.K. Bronich, A. Nehls, A. Eisenberg, V.A. Kabanov, A.V. Kabanov, Novel drug delivery systems based on the complexes of block ionomers and surfactants of opposite charge, Colloids Surf., B 16 (1999) 243-251.

[48] D.M. Mock, G. Lankford, P. Horowitz, A study of the interaction of avidin with 2-anilinonaphthalene-6-sulfonic acid as a probe of the biotin binding site, Biochim. Biophys. Acta 956 (1988) 23-29.

[49] S. Stolnik, L. Illum, S.S. Davis, Long circulating microparticulate drug carriers, Adv. Drug. Del. Rev. 16 (1995) 195-214.

[50] V.P. Torchilin, In vitro and in vivo availability of liposomes, in: A.V. Kabanov, P.L. Felgner, L.W. Seymour (Eds.), Selfassembling Complexes For Gene Delivery: From Laboratory To Clinical Trial, John Wiley, Chichester, New York, Weinheim, Brisbane, Singapore, Toronto, 1998, pp. 277293.

[51] A.E. Hawley, S.S. Davis, L. Illum, Targeting of colloids to lymph nodes: influence of lymphatic physiology and colloidal characteristics, Adv. Drug. Delivery Rev. 17 (1995) 129-148.

[52] R.M. Schiffelers, I.A. Bakker-Woudenberg, S.V. Snijders, G. Storm, Localization of sterically stabilized liposomes in Klebsiella pneumoniae-infected rat lung tissue: influence of liposome characteristics, Biochim. Biophys. Acta 1421 (1999) 329-339.
[53] O. Ishida, K. Maruyama, K. Sasaki, M. Iwatsuru, Sizedependent extravasation and interstitial localization of polyethyleneglycol liposomes in solid tumor-bearing mice, Int. J. Pharm. 190 (1999) 49-56.

[54] G. Kong, R.D. Braun, M.W. Dewhirst, Hyperthermia enables tumor-specific nanoparticle delivery: effect of particle size, Cancer Res. 60 (2000) 4440-4445.

[55] K. Ogawara, M. Yoshida, K. Furumoto, Y. Takakura, M. Hashida, K. Higaki, T. Kimura, Uptake by hepatocytes and biliary excretion of intravenously administered polystyrene microspheres in rats, J. Drug Target. 7 (1999) 213-221.

[56] A.V. Kabanov, Taking polycation gene delivery systems from in vitro to in vivo, Pharm. Sci. Tech. Today 2 (1999) 365-372.

[57] A. Islam, K.S.J. Thompson, S. Akhtar, S.L. Handley, Enhancing antisense oligonucleotide efficacy in vivo by improving both antisense design using RNAse $\mathrm{H}$ accessibility mapping and sustained hippocampal delivery using biodegradeable microspheres, in: Proc. Int. Symp. Controlled Release Bioact. Mater, 27th, 2000, pp. 17-18.

[58] C.E. Harris, S. Agarwal, P.C. Hu, E. Wagner, D.T. Curiel, Receptor-mediated gene transfer to airway epithelial cells in primary culture, Am. J. Respir. Cell Mol. Biol. 9 (1993) 441-447.

[59] S.D. Putney, J. Brown, C. Cucco, R. Lee, T. Skorski, C. Leonetti, T. Geiser, B. Calabretta, G. Zupi, G. Zon, Enhanced anti-tumor effects with microencapsulated c-myc antisense oligonucleotide, Antisense Nucl. Acid Drug Dev. 9 (1999) 451-458.

[60] J.A. Hughes, A.V. Avrutskaya, K.L. Brouwer, E. Wickstrom, R.L. Juliano, Radiolabeling of methylphosphonate and phosphorothioate oligonucleotides and evaluation of their transport in everted rat jejunum sacs, Pharm. Res. 12 (1995) 817-824.

[61] D.W. Miller, A.V. Kabanov, Potential applications of polymers in the delivery of drugs to the central nervous system, Colloids and Surfaces B: Biointerfaces 16 (1999) 321-330.

[62] B. Ji, W. Chen, D.R. Lu, D.S. Halpern, Cell culture and animal studies for intracerebral delivery of borocaptate in liposomal formulation, Drug Deliv. 8 (2001) 13-17.

[63] J. Kreuter, Nanoparticulate systems for brain delivery of drugs, Adv. Drug Deliv. Rev. 47 (2001) 65-81.

[64] W.M. Pardridge, J. Eisenberg, J. Yang, Human blood-brain barrier transferrin receptor, Metabolism 36 (1987) 892-895.

[65] D.W. Miller, B.T. Keller, R.T. Borchardt, Identification and distribution of insulin receptors on cultured bovine brain microvessel endothelial cells: possible function in insulin processing in the blood-brain barrier, J. Cell. Physiol. 161 (1994) 333-341.

[66] W.A. Banks, S.A. Farr, W. Butt, V.B. Kumar, M.W. Franko, J.E. Morley, Delivery across the blood-brain barrier of antisense directed against amyloid beta: reversal of learning and memory deficits in mice overexpressing amyloid precursor protein, J. Pharmacol. Exp. Ther. 297 (2001) 11131121. 